\title{
Vestibular function in the space environment
}

\author{
R. J. VON BAUMGARTEN $\dagger$ \\ University of Mainz, Mainz, G.F.R. \\ G. BALDRIGHIł \\ MHRI University of Michigan, Ann Arbor, Michigan, U.S.A. \\ G. L. SHILLINGER, JR.\& \\ NASA-Ames Research Center, Moffett Field, California, U.S.A.

\section{O. HARTH" AND R. THUEMLER*} \\ University of Mainz, Mainz, G.F.R. \\ (Received 15 July 1974)
}

\begin{abstract}
Adaptation to the weightless state and readaptation after space flight to the 1-G environment on the ground are accompanied by various transitory symptoms of vestibular instability, kinetosis, and illusory sensations. Aside from the problem of how to treat and if possible prevent such symptoms, they offer a clue to a better understanding of normal vestibular functions. Weightlessness is a powerful new "tool" of vestibular research.

Graybiel reported as early as 1952 that human subjects observed the illusion that a real target and the visual afterimage seemed to raise in the visual field during centrifugation when the subjects were looking toward the axis of rotation (oculogravic illusion). In aircraft parabolic-flight weightlessness, human subjects observed that fixed real targets appeared to have moved downward while visual afterimages appeared to have moved upward (oculoagravic illusion).

It can be shown by electronystagmography as well as by a method employing double afterimages that part of this illusion is caused by eye movements that are triggered by the changing input from the otolith system. Another part of the illusion is based on a change of the subjective horizontal and must be caused by convergence of vestibular and visual impulses "behind" the eyes. This part was measured independently of the first one by using a new method. Eye movements could be prevented during these experiments by optical fixation with the right eye on a target at the end of a 24-in. long tube which was rigidly attached paralle] to the longitudinal axis of an aircraft. At the same time the subject tried to line up a shorter tube, which was pivoting around his left eye, with the subjective horizon.
\end{abstract}

\section{Introduction}

THE EVOLUTION of life on this planet took place within the ubiquitous Earthgravitational field. The organic systems of the vertebrates developed in a way that allowed them to overcome the problems imposed by the powerful force of gravity.

\footnotetext{
$\dagger$ Director, Department of Physiology.

$\ddagger$ Research Associate.

\$Research Scientist.

Head Section Biophysic.

"Scientific Assistant.
} 
In the stretch muscles, for instance, a system of monosynaptic proprioceptive reflexes developed, which helped to counteract the continuous downward pull of gravity on the body and eventually allowed the erect posture and gait of man. The cardiovascular system developed new reflexes aimed at adjusting the blood distribution when a large fraction of the total blood volume is pulled into the lower part of the body. The skeleton developed the necessary mechanical strength by incorporation of minerals so it could withstand all the forces imposed while standing and during locomotion within the "ocean" of gravity.

The vestibular system in the inner ear is not made to counteract or to adjust to gravity, but to take advantage of it. The otolith organ is an essential part of the vestibular system, developed to measure one parameter only: the direction in which gravity pulls. It gives to the animal and to humans information about the direction of up and down. This information provides the individual with a frame of reference in space and is important for maintaining equilibrium, the recognition of uphill and downhill, the tilt of objects and many other orientational problems.

Three preconditions are essential for proper functioning of the otolith system:

(1) A gravitational field must be present.

(2) This field must be of the right strength; i.e. $9.81 \mathrm{~m} / \mathrm{sec}^{2}$.

(3) No other forces must be interfering with the gravitational force.

All three conditions were met during evolutionary times and still are met most of the time in normal everyday life. They are not always met, however, in modern life when traveling in cars, boats, or airplanes. Inertial reactive forces frequently occur and interfere with the normal function of the gravity sensors in the otolith system, which causes illusory sensations and sometimes kinetosis, such as car-, sea- or airsickness.

In space flight the otolith system is completely deprived of its adequate stimulus, namely, gravity, and depends solely on the "wrong stimuli"; i.e. inertial reactive forces caused by head movements and locomotion of the crew members. On the Moon and on other planets gravity is present, but being of lesser or greater magnitude than on Earth, it causes "wrong stimuli" to the otolith system, and therefore illusory sensations are again likely to occur.

Fortunately, the principle of redundance, which has proven to be so important for modern space technology, was already in use millions of years ago during the evolution of animals and men. The functions of the vestibular system, for instance, can be taken over to a great extent by the visual system of the eyes, and vice versa, as we know happens in individuals without a vestibular system or who are blind. Only a failure of both systems renders an individual helpless. How well the astronauts could perform in weightlessness and on the Moon came as a great surprise to many vestibular physiologists. The correct information about the astronauts' body positions, in these cases, was supplied mostly by the eye, rather than by the otolith organ.

However, there are still many vestibular problems to be resolved in space flight, especially in regard to long-range missions such as in Skylab or in future flights to other planets. We have learned from the last two Skylab missions that one of the most important and intriguing problems is the intrinsic mechanisms of 
long-term adaptation to the weightless environment and the readaptation to terrestrial conditions after the flight. Another problem is brought about by the desirability of improving the methods of selecting and training the astronauts relative to their individual resistance to vestibular spacesickness. A third problem concerns the best possible use of the new "tool" of weightlessness for a better understanding of the basic physiological mechanisms of the vestibular system. All three problems are closely interrelated.

Former studies of our American-German group were concerned with the behavior and eye movements of unrestrained and restrained fish during horizontal linear acceleration and parabolic flight[1]. This paper will present new results about the interdependence of optical illusory sensations and eye movements in man[2].

Graybiel[3] observed as early as 1952 that human subjects on centrifuges reported the illusion that the image of a real target and the visual afterimage of a real target seemed to raise in the visual field when the subjects were looking toward the axis of the rotating centrifuge. Graybiel named this illusion the "oculogravic illusion," which is based on an increase in magnitude and a change in the direction of the gravitoinertial vector. In 1958 Gerathewohl and Stallings [4] observed a related illusion during parabolic flight in jet aircraft, which they called oculoagravic illusion. During the state of weightlessness the visual afterimage of a target seemed also to raise in the visual field. In addition, Roman and coworkers [5] found in later parabolic flight that while the visual afterimage raises, at the same time a real target appears lowered in the visual field in contrast with the oculogravic illusion reported on the ground (Fig. 1).

To establish to what degree these illusions can be explained by eye movements and to what degree by neuronal integration in the brain, our group tried to measure real eye movements as they occur in the dark without optical fixation, during rectilinear accelerations on the ground, and during weightlessness in parabolic flight.

\section{Methods and results}

One series of experiments was performed in a rapidly accelerating and braking race car on the runway of an airfield. Since a greater acceleration was obtained by braking the car than by forward acceleration, the braking action of the car was

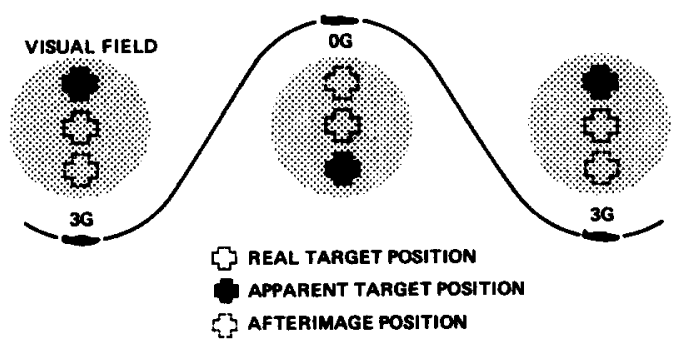

Fig. 1. Apparent position of a real target and an afterimage during a parabolic maneuver. (From Clark [6].) 
mostly used for the experiments. To avoid optical fixation the subjects wore darkened skindiver goggles but were asked to keep their eyes open in the dark during the experiment. The head was fixed in a rigid headholder. The eye movements were recorded electronystagmographically using pure unfiltered d-crecording (Fig. 2a). Forward acceleration caused a downward rotation of the eyeballs. During backward acceleration (Fig. 2b) the eyeballs rotated upward. When, in other experiments, a target represented by a miniature lightbulb was fixated during the runs, and a visual afterimage was produced by a photoflash at the level of the target before the run, the target and the visual afterimage seemed to raise together, without separation, in the visual field. This is comparable to the oculogravic illusion. When the subject tried not to fixate the target, but his own visual afterimage, the afterimage separated from the target during forward acceleration and moved downward, while the target seemed to move in the opposite direction. The separation of the target from the visual afterimage indicates movement of the eye which can be prevented only by visual fixation of the target.

(a)
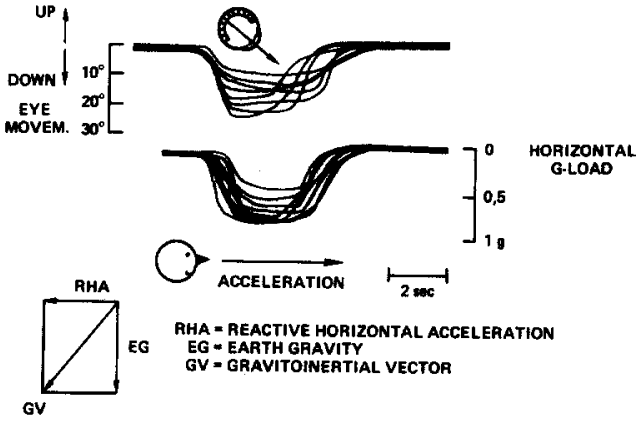

(b)

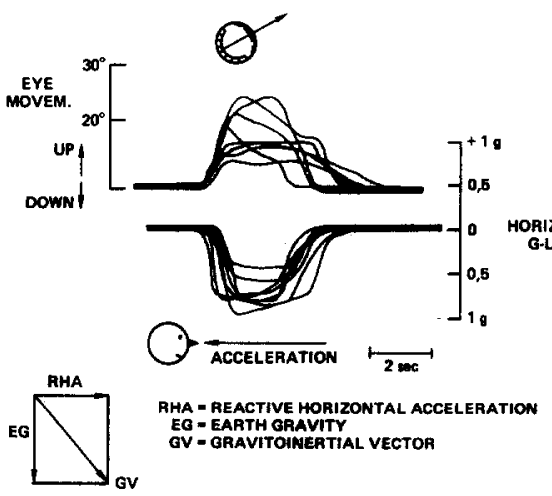

Fig. 2. Effects of forward acceleration on human eye position. Superimposed records of eight consecutive tests with the same subject. Upper trace represents d-c electronystagmogram of eye movements. Lower trace is the $\boldsymbol{x}$-axis $\mathrm{G}$ load. Bottom-of-picture insert shows vectorial forces acting on statoconial membrane.

(a) Forward acceleration. Note downward deflection of the eyes.

(b) Backward acceleration. Note upward deflection of the eyes. 
Next we studied the eye movements related to the oculoagravic and oculogravic illusions during parabolic aircraft flight. Most experiments were conducted in a Lear Jet aircraft at NASA-Ames Research Center. After we learned that human eye movement occurs as rapidly as within one second, more experiments were conducted in a light propeller-driven aircraft in Mainz, Germany. The experimental set-up was similar to the one used in the race car, and electronystagmography was again used to record the eye movements. The test pilots were asked to maintain the acceleration in the longitudinal axis ( $x$-axis) at zero during the maneuver by using the throttle and observing the accelerometers in the cockpit, but to change the normal ( $z$-axis) acceleration to prescribed values by pulling or pushing the control column [7]. Due to the speed of the aircraft the angular acceleration during parabolic flights could be kept below threshold for stimulation of the semicircular canal system. During the initial pullup, when acceleration values higher than $1 \mathrm{G}$ were reached, the eyeballs of the human subjects rotated with the line of sight to a downward position (Fig. 3). After pushover, when weightlessness was obtained, the eyeballs rotated to an upward position and stayed there until the pullout, when the eyes rotated again downward. The eyes returned again to the control position during normal flight. Small nystagmus occurred occasionally with the fast phase downward when the eyes reached an upward position and with the fast phase upward when the eyes reached a downward position. These small deflections support our view that the tracings we obtained are genuine physiological recordings and not amplifier drifts or drifts due to the electrode contact potentials. However, the possibility of such drifts with d-c electronystagmography can never be completely excluded, especially under the conditions of parabolic flight when the subject sweats and the pressure of the electrodes on the skin changes during pullup, pushover, and pullout.

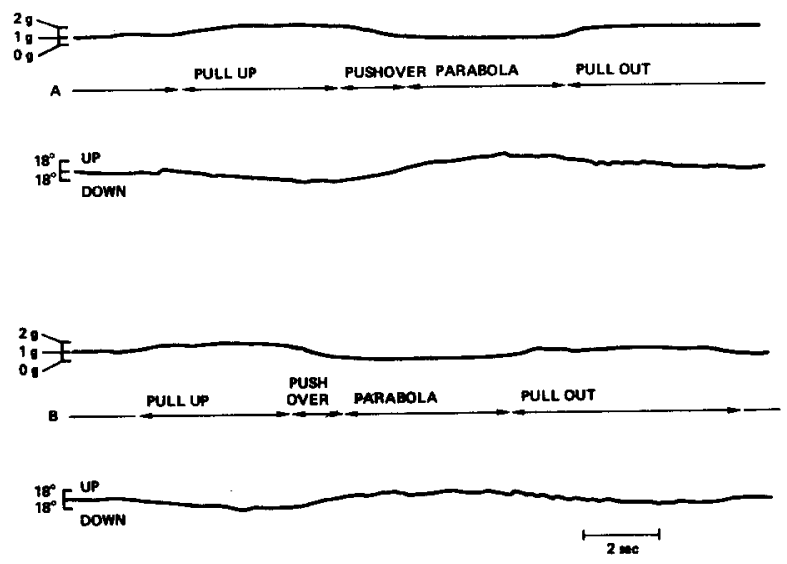

Fig. 3. Eye movements during parabolic flight. $A$ and $B$ show similar results from two consecutive runs of the same experiment. Upper trace, $z$-axis $G$ load. Lower trace, electronystagmogram of eye movements. High-G load causes downward eye deflections. Low-G load and weightlessness cause upward eye deflections. Note the reversal of nystagmus between low- and high-G loads. 
Therefore we decided to confirm our results by measuring the eye deflections with a different method. We used a technique similar to one already described by Niven et al. [8] in a paper about illusions observed in elevators. This method allows us to measure eye movements very precisely by using double afterimages. The head of the subject in our experiments was fixed within the airplane in a rigid headholder (Fig. 4). The subject looked straight ahead through a light-tight-sealed skindiver mask into a 24-in. tube. At the other end of the tube, two photoflashes were attached side by side with a small target light between them. A pulsating light source helped to maintain the afterimages over periods exceeding one minute.

During normal flight (Fig. 5) the target light was switched on and the left flash was triggered. Next, the target light was switched off and the pilot started to fly a parabolic trajectory. When weightlessness was obtained, the right flash was triggered. Next, the blinking light was switched on, and the subject reported in a tape recorder the exact position of the two afterimages as seen on a graduated screen. All eight subjects reported the position of the afterimage from the second flash in a position 2 to $8^{\circ}$ lower in the visual field than that of the first flash. These observations can be explained only by an upward rotation of the eyeballs when the $\mathbf{G}$ force is less than one or during weightlessness.

When the first fiash was triggered in normal flight and the second flash during the high-G pullup maneuver, the afterimage of the second flash was seen in a higher position than the afterimage of the first flash, indicating a downward deflection of the eye.

The question arises as to how such eye movements would affect the apparent position of a real fixed target (Fig. 6). During the pullup the eyes moved with the line of sight downward. A real fixed target in front of the observer is in normal flight projected to the middle of the retina. After the eye moves downward during transition to a higher than 1-G load the target is projected to the lower part of the retina and consequently seen in the corresponding upper part of the visual field. This corresponds well with the oculogravic illusion reported by Graybiel[3].

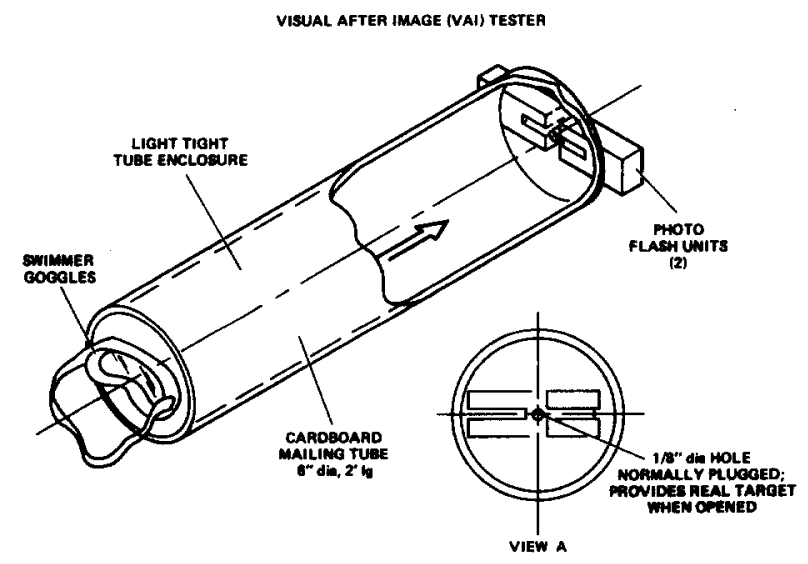

Fig. 4. Set-up to measure eye movements by the use of two consecutive afterimages. Description in the text. 


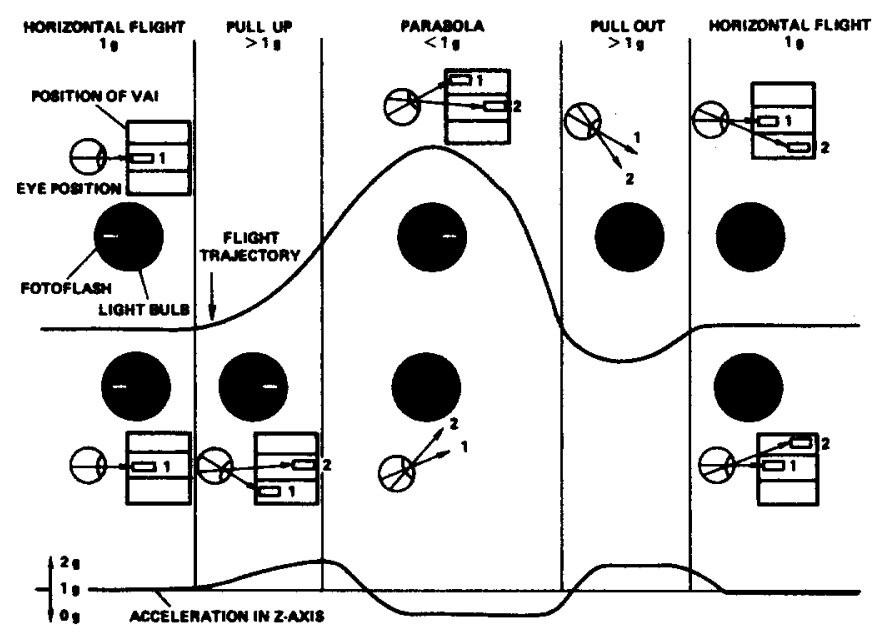

Fig. 5. Eye movements during parabolic flight as detected by the visual afterimage (VAI) method. The figure shows from left to right events at different phases of parabolic flight. Upper part: During normal flight the subject fixates on a miniature light bulb and obtains a VAI by photoflash in the left visual field. During the parabola, at $0 \mathrm{G}$, a second photoflash is given in the right half of the visual field at the same level of the first. Since the eyes rotate upward during $0 \mathrm{G}$, the second VAI is seen separated and lower than the first one. During the pullup phase, higher than $1 \mathrm{G}$ load, the eyes and both VAIs rotate to a downward position while the separation of the VAI is maintained.

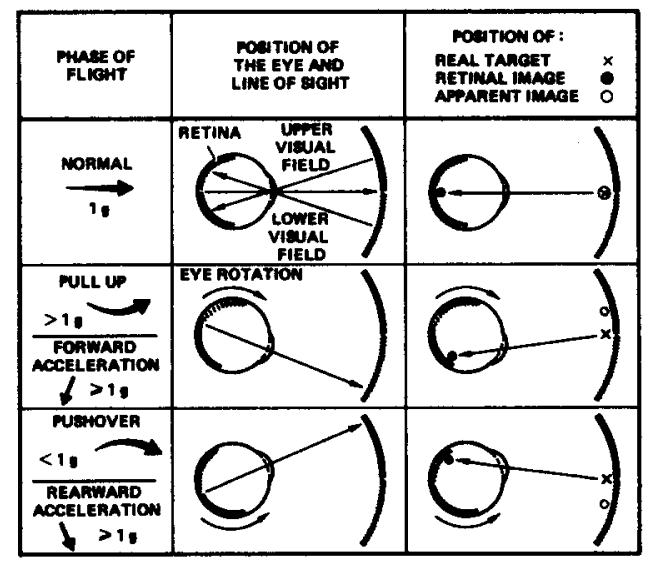

Fig. 6. Visual response to different G-forces in man. The figure illustrates the dependence of retinal and apparent images on the eye movements caused by horizontal acceleration and parabolic flight. During increased G-force (pullup and pullout) the eyes rotate downward; consequently, the retinal image must fall in the lower part of the retina which corresponds to the upper part of the visual field. During lowered G-forces (pushover) the eyes rotate upward and the shift of the image is inverted as compared to the pullup and pullout. 
During the pushover, the eyes move with the line of sight upward. The fixed target would then be projected to the upper part of the retina, which corresponds to the lower part of the visual field. This would agree well with the oculoagravic illusion reported by Gerathewohl and Stallings [4].

The occurrence of predictable eye movements during parabolic flight does not exclude the possibility that a fraction of the oculogravic and oculoagravic illusions may be caused by a nervous convergence of vestibular and visual impulses "behind" the eye, as originally thought by Graybiel. In order to measure this part of the illusion separately from the part caused by eye movements, we had to develop a new technique. The problem was to prevent any eye movements during the test flight while fixating a fixed target, but at the same time to reset such a target to the subjective horizon after it appeared to have moved, without causing any eye or head movements by the resetting process. The problem was solved by using one eye for fixation and the other eye for the resetting process. The subject was asked to strongly fixate with the right eye throughout the experiment on the light appearing through the translucent end of the long tube which was rigidly fixed parallel to the longitudinal axis of the airplane (Fig. 7). At the same time the subject looked with the left eye into a shorter tube whose far end could be moved up or down by the subject. During transition to the high-G pullup both tubes seemed to move up. They seemed to move down during the diminishing-G pushover. The subject was asked to manually keep the short tube always lined up with the subjective horizon, while still fixating the target in the long tube. Because both eyes are always yoked together in their actual positions, any eye movements could be excluded in this experiment. The deflections of the short tube activated an electrical potentiometer, and the corresponding output was recorded by direct-writing oscillograph. In this manner a record of the angular position of the short tube was obtained. Figure 8 shows that, during transition to zero $G$, the short tube was actively moved upward by the subject compensating for an apparent downward movement of the fixed target. During higher than $1-G$ loads which occur at the

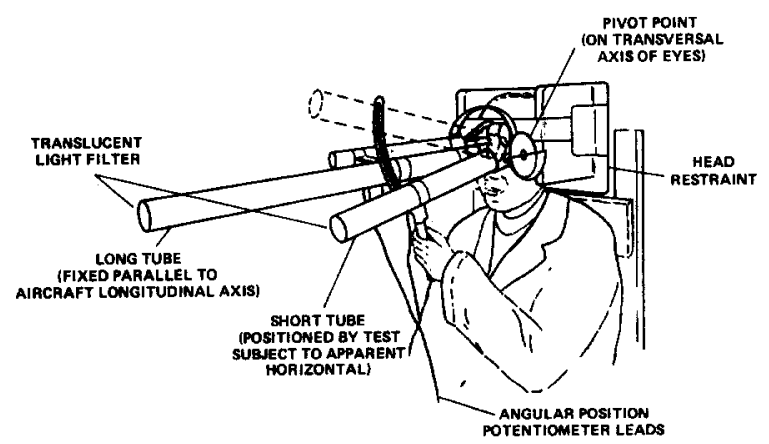

Fig. 7. Double-tube setup for measurement of apparent target shift independently of eye movements. The long tube is rigidly mounted parallel to the longitudinal axis of the aircraft. The short tube can pivot on the left eye so that its far end moves up or down. The subject is asked to maintain optical fixation on the translucent end of the long tube while lining up the short tube with the subjective horizon. The deflections of the short tube activate an electrical potentiometer circuit, the output of which is then recorded as angular position. 


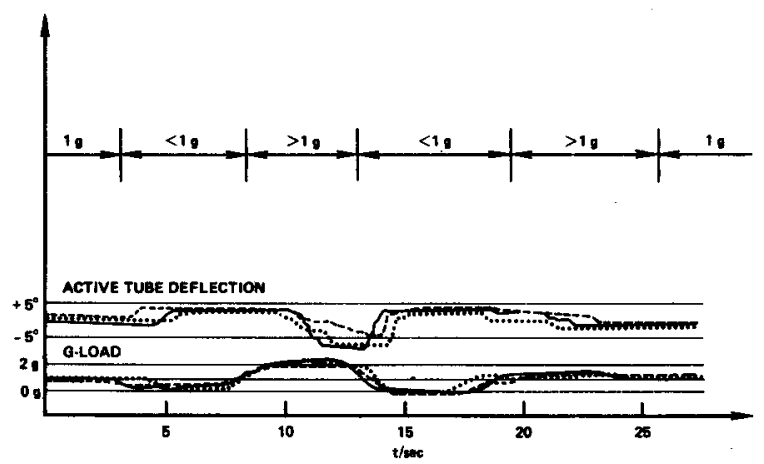

Fig. 8. Oculogravic and oculoagravic illusions measured while the eyes were fixating in parabolic flight. The upper traces illustrate the estimated flight trajectory of the aircraft. The traces in the middle indicate active deflections of the shorter tube caused by the subject trying to keep it lined with the subjective horizon. The lower traces represent the $z$-axis $\mathbf{G}$ load as recorded by an electrical accelerometer.

pullup and pullout, the short tube was actively moved downward, compensating for an apparent upward movement of the fixated target.

\section{Conclusions}

Results from these experiments correspond well with the oculogravic and oculoagravic illusions. The new contribution is that now it is possible to measure which part of the illusions is caused by the optic within the eye and which part by convergence of vestibular and visual impulses "behind" the eye; i.e. those centers of the brain that are concerned with body position. Additional work is needed to obtain quantitative data and to compare the two parts of the illusion with the subject at different tilt angles and body positions according to a scheme given by Schöne [9] and by Correia et al.[10].

Space-related vestibular research has gone from the period when it tried to assist in the planning and preparation of humans for space flight to the stage in which data from weightlessness flights are beginning to improve our understanding of the vestibular system. Long-term weightlessness as available in space flight is a powerful and promising new tool for the vestibular physiologist.

Acknowledgment-This work was supported by NASA contract No. NAS 2-6057 NASA-Ames Research Center, Moffett Field, California.

\section{References}

1. Von Baumgarten, R. J., Baldrighi, G., Atema, J. and Shillinger, G. L., Jr., Behavioral responses to linear accelerations in blind goldfish, Space Life Sciences 3, 25-33 (1971).

2. Von Baumgarten, R. J., Baldrighi, G. and Shillinger, G. L., Jr., Vestibular behavior of fish during diminished G-force and weightlessness, Aerospace Med. 43, 626-632 (1972).

3. Graybiel, A., Oculogravic illusion, Arch. Ophthalmol. 48, 605-615 (1952).

4. Gerathewohl, S. J. and Stallings, H. D., Experiments during weightlessness: A study of the oculoagravic illusion, J. Aviat. Med. 29, 504-516 (1958).

5. Roman, J. A., Warren, B. H. and Graybiel, A., Observations of the elevator illusion during subgravity preceded by negative accelerations, Aerospace Med. 35, 121-124 (1964). 
6. Clark, B., Visual space perception as influenced by unusual vestibular stimulation, Human Factors 5, 265-274 (1963).

7. Shillinger, G. L., Jr., Von Baumgarten, R. J. and Baldrighi, G., The gravity reference response, the rotation sensation, and other illusory sensations experienced in aircraft and space flight, Space Life Sciences (1973, in press).

8. Niven, J. I., Whiteside, T. C. D. and Graybiel, A., The elevator illusion: Apparent motion of a visual target during vertical acceleration, Nav. Sch. Aviat. Med. Rpt., NSAM-876, Flying Personnel Res. Committee Rpt., 1213, NASA Order R-93, Pensacola, Fla., and Air Ministry London (1963).

9. Schöne, $H$., On the role of gravity in human spatial orientation, Aerospace Med. 37, 764-772 (1964).

10. Correia, M. J., Hixson, W. C. and Niven, I., Otolith shear and the visual perception of force direction: Discrepancies and proposed resolution, Nav. Aerospace Med. Inst. Rpt., NAMI-951, Pensacola, Fla. (1965). 